\title{
Classification of Ordered Texture Images using Regression Modelling and Granulometric Features
}

\author{
Mahmuda Khatun \\ Department of Mathematics \\ and Statistics \\ University of Strathclyde \\ Glasgow, UK G1 1XH \\ Email:m.khatun@strath.ac.uk
}

\author{
Alison Gray \\ Department of Mathematics \\ and Statistics \\ University of Strathclyde \\ Glasgow, UK G1 1XH \\ Email: a.j.gray@strath.ac.uk
}

\author{
Stephen Marshall \\ Department of Electronic \\ and Electrical Engineering \\ University of Strathclyde \\ Glasgow, UK G1 1XW \\ Email: s.marshall@eee.strath.ac.uk
}

\begin{abstract}
Structural information available from the granulometry of an image has been used widely in image texture analysis and classification. In this paper we present a method for classifying texture images which follow an intrinsic ordering of textures, using polynomial regression to express granulometric moments as a function of class label. Separate models are built for each individual moment and combined for back-prediction of the class label of a new image. The methodology was developed on synthetic images of evolving textures and tested using real images of 8 different grades of cut-tear-curl black tea leaves. For comparison, grey level co-occurrence (GLCM) based features were also computed, and both feature types were used in a range of classifiers including the regression approach. Experimental results demonstrate the superiority of the granulometric moments over GLCM-based features for classifying these tea images.
\end{abstract}

Index Terms-Granulometry, pattern spectrum, structuring element, ordered texture, tea granule images

\section{INTRODUCTION}

Many methodologies have been proposed to determine the physical/chemical properties of foods, using chemometrics, pattern recognition and/or image analysis techniques, e.g. [1][8]. Applications of digital image processing techniques are expanding rapidly in the food processing industries.

Here we investigate the advantages of granulometric moments over GLCM-based features for classifying 8 different grades of tea images. Granulometries extract shape-based textural information, hence are useful for analysing and classifying shape-based images. Originally developed by Matheron [9] in the binary case to characterise the size and shape information of a random set, the granulometric approach was extended to the grey scale case by many others, e.g. [10][14]. It has been used extensively in texture analysis and classification. For example, granulometric moments were used to characterise evolution of a dynamic process concerning paint drying in [15]. Binary images of the corneal endothelium were classified as normal or pathological cases in [16] using the granulometric size distribution of the images. The first two granulometric moments were used to classify white blood cells in bone marrow images in [17]. Evolving texture images of corrosion were classified using granulometric moments by means of a parallel evolution function in [18]. A combination of opening granulometry and closing granulometry was em- ployed in [19] to classify grey scale Brodatz texture images [20].

GLCM is a statistical approach to texture classification which characterises the spatial relationships between the grey levels of pixels, and has proved useful in various texture classification applications because of its ability to extract spatial information. GLCM features were used in a selforganising map in [21] to classify Brodatz texture images with $97 \%$ classification accuracy. GLCM features and linear discriminant analysis (LDA) were successfully used to classify colour images of colon cancer in [22]. These features were also found to be useful for classifying colour texture images in [23].

In this work, we consider ordered textures. We define ordered textures as those which can be ranked according to some scale of fineness or coarseness, or by size of texture primitives (shapes) in the images. Building on [18], we develop a regression-based classifier by modelling granulometric moments or GLCM features as a function of texture class label. A cubic polynomial regression is fitted for each chosen feature separately, and then a combined cubic polynomial regression is obtained for back-prediction of the class label of a new image using its observed features. Several classifiers, i.e. a support vector machine (SVM), LDA, a feed-forward neural network (FF-NNET) and the regression classifier were employed using the same sets of features to compare their relative classification accuracy.

For testing, we use a sequence of cut-tear-curl (CTC) black tea images (Figure 1) representing 8 different grades of tea of different granule sizes as used in [5]. The images were sorted visually according to their granule size and were labelled as class 1 to 8 , from the smallest to the largest granule size, so that the tea granules increase in size between the classes. The next section briefly describes the methods used in [5], with some other work relating to tea images.

\section{A. Related work}

Sorting of tea into different grades according to granule size is a very important task in the tea processing industry. This has traditionally been carried out by sieving with a series of sieves of differently sized mesh, however recently some researchers 
have investigated texture analysis and classification techniques to develop a more automated approach. Some recent work is summarised here.

Indian CTC black tea granules comprising eight grades of tea from different tea gardens in Assam, India were classified according to granule size in [5]. Four-level pyramidal decomposition using Daubechies wavelets was applied to each grey scale image. Initially, energy and entropy from each of four approximation sub-bands were used as features in principal component analysis (PCA) and the self-organising map (SOM) clustering technique, however these were unable to clearly separate the grades. A pair of most similar images from each grade were identified using Mahalanobis distance of their feature vectors, and one of these images was selected as a typical image from that grade. New features were calculated as the Mahalanobis distance of a given image to the chosen typical image in each grade. With the new distance features, PCA and SOM were able to distinguish the grades better. Two different neural networks, a multi-layer perception (MLP) and learning vector quantisation (LVQ), were trained using 700 images and tested on another 150 images, and achieved $74.67 \%$ and $80 \%$ classification accuracy respectively. In this paper, we apply our methodology to some of these same images below, with much improved results.

Hyperspectral images of five grades of roasted green tea leaves were classified in [4]. Four texture descriptors, namely mean, sd, energy and entropy, were computed from 3 optimum waveband images (chosen by PCA), and used as features in a SVM, with $95 \%$ correct classification. In [7], six different classes of tea were used. The discrete cosine transform was used on multi-spectral images (red, near-infrared (NIR) and green bands). Using the standard deviation (sd) of each of the original or filtered NIR images with a SVM produced $73.33 \%$ or $100 \%$ correct classification respectively. Five different grades of Chinese green tea brands were classified using multi-spectral colour images in [8]. GLCM features computed from wavelet decomposition of each of the three image colour planes were used with LDA, giving $100 \%$ accurate classification. Four different categories of Chinese green tea were distinguished using entropy values calculated from multispectral images in [6]. A least squares support vector machine produced $97.5 \%$ to $100 \%$ classification accuracy.

\section{Methodology}

Texture classification involves a step to extract features from the image under study, and a classification step, in which a texture class membership is assigned to the image, based on information provided by the extracted texture features through appropriate machine learning algorithms [24]. This section describes the feature extraction techniques and classifiers used in this work.

\section{A. Opening granulometry}

As a texture image can be considered as a collection of grains [13], the concept of opening granulometry is to sieve the grains through filters of increasing size, so that grains with size smaller than the sieve mesh drop out and only grains with larger sizes remain. The shape of the holes is determined by the shape of the structuring element (SE), which is a geometrical pattern used to extract textural information from a given digital image [25]. That is, if image $f$ is opened sequentially by a series of SEs of increasing size, $\left\{g_{1}, g_{2}, \ldots, g_{N}\right\}$, at each stage of opening the finer details will successively be eliminated and the volume (sum of pixel intensities) of the input image will reduce eventually to zero, i.e. $\Omega(1) \geq \Omega(2) \geq \ldots \geq \Omega(N)$, where $\Omega(j)$ is the image volume left after the $j^{\text {th }}$ opening. This decreasing sequence is called the size distribution [13].

Normalising the size distribution as $\Phi(n)=1-\Omega(n) / \Omega(0)$, where $\Omega(0)$ is the original image volume, gives a cumulative distribution function (cdf) which rises monotonically from 0 to 1 as the size of the SE increases. Its derivative $\Phi^{\prime}(n)=d \Phi(n) / d n$ is a probability density function (pdf), referred to as the pattern spectrum (PS) [26]. As a pdf, this can be summarised by its statistical moments. In the discrete case, the scaling factor $n$ is an integer, so the $m^{\text {th }}$ granulometric moment may be calculated as

$$
\mu_{m}=\sum_{n=1}^{N} n^{m} \Phi^{\prime}(n) .
$$

We use these moments to compute the mean $\mu=\mu_{1}$, standard deviation (sd) $\sigma=\sqrt{\nu_{2}}$, skewness and (excess) kurtosis of $\Phi^{\prime}(n)$, calculated from the central moments $\nu_{m}=$ $\sum_{n=1}^{N}\left(n-\mu_{1}\right)^{m} \Phi^{\prime}(n)$. The skewness is $\nu_{3} / \sigma^{3}$, and kurtosis is $\nu_{4} / \sigma^{4}-3$. We refer to these four moments below as the PS moments. These moments contain useful textural information to characterise the texture images.

\section{B. Grey level co-occurrence matrix (GLCM)}

The GLCM is a $G \times G$ matrix where $G$ is the number of grey levels in the original image. The entry $C(i, j)$ of a GLCM is the frequency of grey levels $i$ and $j$ of pixels separated by inter-pixel distance $d$ and lying on a line at angle $\phi$ to the reference direction of the image. To reduce the sparsity of the GLCM, the original image is often quantised first to some lower level, e.g. 8, 32, or 64 [27]. The normalised GLCM $p(i, j)$ can be obtained by dividing $C(i, j)$ by the sum of its entries as

$$
p(i, j)=C(i, j) / \sum_{k=1}^{G} \sum_{l=1}^{G} C(k, l),
$$

where $G$ is the number of grey levels after any quantisation.

Haralick et al. [28] proposed extracting some features from the GLCM for more compact texture representation, including:

1) Maximum probability $: \max _{(i, j)} p(i, j)$

2) Energy : $\sum_{i=1}^{G} \sum_{j=1}^{G} p(i, j)^{2}$

3) Entropy : $-\sum_{i=1}^{G} \sum_{j=1}^{G} p(i, j) \log p(i, j)$

4) Contrast: $\sum_{i=1}^{G} \sum_{j=1}^{G}(i-j)^{2} p(i, j)$

5) Homogeneity: $\sum_{i=1}^{G} \sum_{j=1}^{G} \frac{p(i, j)}{1+|i-j|}$ 
6) Correlation : $\frac{1}{\sigma_{i} \sigma_{j}} \sum_{i=1}^{G} \sum_{j=1}^{G}\left(i-\mu_{i}\right)\left(j-\mu_{j}\right) p(i, j)$, where $\mu_{i}=\sum_{i=1}^{G} i \sum_{j=1}^{G} p(i, j), \mu_{j}=\sum_{i=1}^{G} j \sum_{j=1}^{G} p(i, j)$, $\sigma_{i}^{2}=\sum_{i=1}^{G}\left(i-\mu_{i}\right)^{2} \sum_{j=1}^{G} p(i, j)$, and $\sigma_{j}^{2}=\sum_{j=1}^{G}(j-$ $\left.\mu_{j}\right)^{2} \sum_{i=1}^{G} p(i, j)$.

We also use these below as texture features for classification.

\section{Classifiers}

Let $Y_{i}(c), i=1,2, \ldots, p$, and $c=1,2, \ldots, K$ be the average value of the $i^{t h}$ feature for the images in the $c^{\text {th }}$ class. A regression classifier is built by modelling each average feature separately as a function of class label using a cubic polynomial regression model, written as:

$$
Y_{i}(c)=\beta_{0}^{(i)}+\beta_{1}^{(i)} * c+\beta_{2}^{(i)} * c^{2}+\beta_{3}^{(i)} * c^{3}+\xi_{i},
$$

where the parameters $\beta$ terms are estimated using least squares and the $\xi_{i}$ are error terms.

For a single feature $i$ the model is of the form:

$$
\left[\begin{array}{c}
Y_{i}(1) \\
Y_{i}(2) \\
\vdots \\
Y_{i}(K)
\end{array}\right]=\left[\begin{array}{c}
\beta_{0}^{(i)}+\beta_{1}^{(i)} c_{1}+\beta_{2}^{(i)} c_{1}^{2}+\beta_{3}^{(i)} c_{1}^{3} \\
\beta_{0}^{(i)}+\beta_{1}^{(i)} c_{2}+\beta_{2}^{(i)} c_{2}^{2}+\beta_{3}^{(i)} c_{2}^{3} \\
\vdots \\
\beta_{0}^{(i)}+\beta_{1}^{(i)} c_{K}+\beta_{2}^{(i)} c_{K}^{2}+\beta_{3}^{(i)} c_{K}^{3}
\end{array}\right]+\left[\begin{array}{c}
\varepsilon_{1} \\
\varepsilon_{2} \\
\vdots \\
\varepsilon_{K}
\end{array}\right]
$$

For $p$ such features, a combined fitted model relating each feature to class is formed as:

$$
\left[\begin{array}{c}
\hat{Y}_{1}(c) \\
\hat{Y}_{2}(c) \\
\vdots \\
\hat{Y}_{p}(c)
\end{array}\right]=\left[\begin{array}{c}
\hat{\beta}_{0}^{(1)}+\hat{\beta}_{1}^{(1)} c+\hat{\beta}_{2}^{(1)} c^{2}+\hat{\beta}_{3}^{(1)} c^{3} \\
\hat{\beta}_{0}^{(2)}+\hat{\beta}_{1}^{(2)} c+\hat{\beta}_{2}^{(2)} c^{2}+\hat{\beta}_{3}^{(2)} c^{3} \\
\vdots \\
\hat{\beta}_{0}^{(p)}+\hat{\beta}_{1}^{(p)} c+\hat{\beta}_{2}^{(p)} c^{2}+\hat{\beta}_{3}^{(p)} c^{3}
\end{array}\right]
$$

or $\hat{\mathbf{Y}}=\hat{\mathbf{B} C}$, where

$$
\hat{\mathbf{B}}=\left[\begin{array}{cccc}
\hat{\beta}_{0}^{(1)} & \hat{\beta}_{1}^{(1)} & \hat{\beta}_{2}^{(1)} & \hat{\beta}_{3}^{(1)} \\
\hat{\beta}_{0}^{(2)} & \hat{\beta}_{1}^{(2)} & \hat{\beta}_{2}^{(2)} & \hat{\beta}_{3}^{(2)} \\
\vdots & \vdots & \vdots & \vdots \\
\hat{\beta}_{0}^{(p)} & \hat{\beta}_{1}^{(p)} & \hat{\beta}_{2}^{(p)} & \hat{\beta}_{3}^{(p)}
\end{array}\right]
$$

is a $p \times 4$ matrix and $\mathbf{C}=\left[1, c, c^{2}, c^{3}\right]^{\prime}$ is a $4 \times 1$ vector. This combined model is used for prediction.

The above can be re-written as

$$
\left[\begin{array}{c}
\hat{Y}_{1}(c) \\
\hat{Y}_{2}(c) \\
\vdots \\
\hat{Y}_{p}(c)
\end{array}\right]-\left[\begin{array}{c}
\hat{\beta}_{0}^{(1)} \\
\hat{\beta}_{0}^{(2)} \\
\vdots \\
\hat{\beta}_{0}^{(p)}
\end{array}\right]=\left[\begin{array}{ccc}
\hat{\beta}_{1}^{(1)} & \hat{\beta}_{2}^{(1)} & \hat{\beta}_{3}^{(1)} \\
\hat{\beta}_{1}^{(2)} & \hat{\beta}_{2}^{(2)} & \hat{\beta}_{3}^{(2)} \\
\vdots & \vdots & \vdots \\
\hat{\beta}_{1}^{(p)} & \hat{\beta}_{2}^{(p)} & \hat{\beta}_{3}^{(p)}
\end{array}\right]\left[\begin{array}{c}
c \\
c^{2} \\
c^{3}
\end{array}\right]
$$

or, using matrix notation, as:

$$
\left[\hat{\mathbf{Y}}-\hat{\boldsymbol{\beta}}_{0}\right]=\left[\begin{array}{lll}
\hat{\boldsymbol{\beta}}_{1} & \hat{\boldsymbol{\beta}}_{2} & \hat{\boldsymbol{\beta}}_{3}
\end{array}\right]\left[\begin{array}{c}
c \\
c^{2} \\
c^{3}
\end{array}\right] .
$$

Pre-multiplying by $\left(\hat{\boldsymbol{Y}}-\hat{\boldsymbol{\beta}}_{0}\right)^{\prime}$ gives

$\left(\hat{\mathbf{Y}}-\hat{\boldsymbol{\beta}}_{0}\right)^{\prime}\left(\hat{\mathbf{Y}}-\hat{\boldsymbol{\beta}}_{0}\right)=\left(\hat{\mathbf{Y}}-\hat{\boldsymbol{\beta}}_{0}\right)^{\prime}\left[\begin{array}{lll}\hat{\boldsymbol{\beta}}_{1} & \hat{\boldsymbol{\beta}}_{2} & \hat{\boldsymbol{\beta}}_{3}\end{array}\right]\left[\begin{array}{c}c \\ c^{2} \\ c^{3}\end{array}\right]$,

a cubic equation in $c$ of the form $A_{1} c^{3}+A_{2} c^{2}+A_{3} c+A_{4}=0$. A positive real root of this equation is used as the predicted class $c$. Where there is more than one positive real root we choose the smallest one (as was appropriate in all our training examples). If none of the roots are positive and real the method fails to predict the class, and we choose the first class as the prediction.

We employed SVM, LDA and FF-NNET as benchmark methods to compare the performance of this regression approach.

Developed by Vapnik and Cortes [29], SVM is a very powerful classification technique, which is robust in producing high classification accuracy even in high-dimensional data spaces with non-linearly separable classes [30]. Its theoretical foundation is based on the structural risk minimisation principle [31]. We used the one-to-one classification approach for this multiple class problem, and tuned the choice of kernel, any associated kernel parameter values and the cost parameter used, in the SVM training for optimum classification results. We also used LDA, which assigns a new feature vector to the class with maximum posterior probability, assuming normality of the class conditional distributions and a common withinclass feature covariance matrix [32]. A FF-NNET with single hidden layer is also used [30], with the number of neurons in the hidden layer optimised in training. The $\mathrm{R}$ software package was used for SVM, LDA and FF-NNET with libraries e1071, MASS and nnet respectively.

The prediction abilities of the classifiers are assessed using misclassification rate and mean absolute error (MAE), defined as:

$$
\mathrm{MAE}=\frac{1}{n} \sum_{i=1}^{n}\left|t_{\text {pred }}^{i}-t_{\text {act }}^{i}\right|
$$

where $n$ is the number of images for which the class is to be predicted, and $t_{\text {pred }}^{i}$ and $t_{\text {act }}^{i}$ are respectively the predicted class label (rounded if necessary) and the actual class label of image $i$.

\section{Application to tea images}

\section{A. Data description}

Each image is a colour image of size $2000 \times 3008$. The eight different classes are BOPL (Broken Orange Pekoe Large), BOP (Broken Orange Pekoe), BOPSM (Broken Orange Pekoe Small), BP (Broken Pekoe), PF (Pekoe Fannings), PD (Pekoe Dust), OF (Orange Fannings), and Dust, and the approximate 
diameters in $\mathrm{mm}$ of the granules are $2.0,1.7,1.3,1.0,0.5$, $0.355,0.25$ and Not specific respectively [5].

We obtained training and test sets by extracting $256^{2}$ size images from each of the original images and converted them to grey scale. Fifty non-overlapping sub-images were extracted from one image from each class, giving a total of 400 sample images. One sub-image from each class is shown in Figure 1, to show the progression in size of the tea granules over the classes.

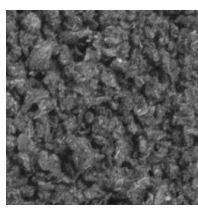

(a) Class 1

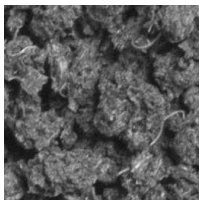

(e) Class 5

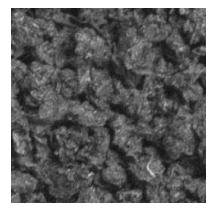

(b) Class 2

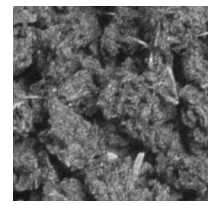

(f) Class 6

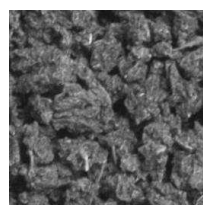

(c) Class 3

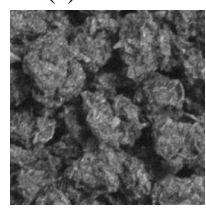

(g) Class 7

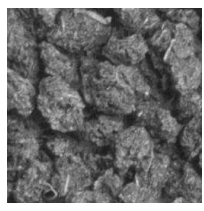

(d) Class 4

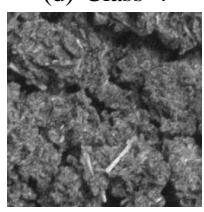

(h) Class 8
Fig. 1: Sample grey scale tea images of size $256^{2}$, one from each of the eight tea classes.

\section{B. Feature extraction}

There exists substantial variation of intensity within images. To improve this, top-hat transformation was applied as a preprocessing step in order to suppress dark parts and highlight bright parts of the images. Since granule size increases over classes, a disk SE of increasing size is used in the top-hat transformation. Then we applied granulometry using a square and a disk SE and computed the first four PS moments of the pattern spectrum from each SE. Average PS moments are obtained using all sub-images from each class and are plotted against class in Figure 2. The PS mean and sd using all SEs increase with class, while skewness decreases, though for the disk SE the decrease is very slow. Kurtosis for both square and disk SEs is negative and increases slightly with class.

These PS moments are then used as texture features for predicting the class of a tea image. We implemented the regression approach (REG), SVM, neural network and LDA as classifiers and compared their efficiency.

We then computed the six different GLCM features in Section II from each of the 50 sub-images from each class at quantisation level 8 for four different orientations and separation 1, and averaged these over all sub-images. The average features are plotted against class in Figure 3. Entropy and correlation increase with class. Maximum probability and energy decrease with class, but no trend is clear for contrast or homogeneity as a function of class.

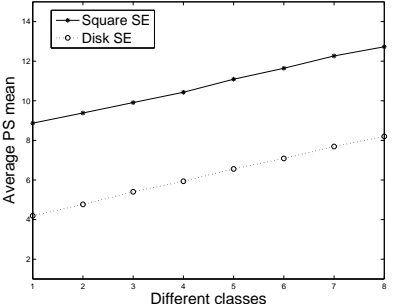

(a) PS mean

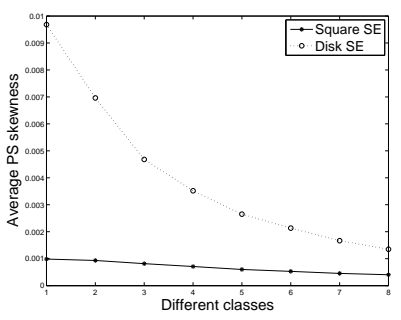

(c) PS skewness

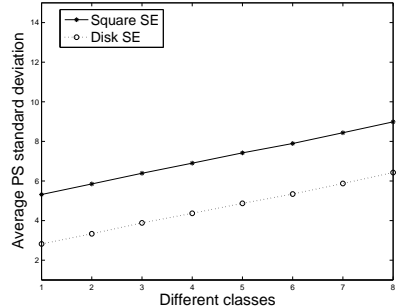

(b) PS sd

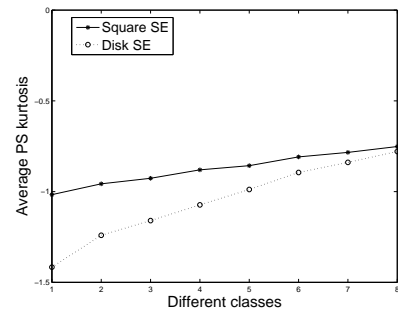

(d) PS kurtosis
Fig. 2: Average PS moments, of all 50 top-hat sub-images from each class, plotted against class, for square and disk SEs.

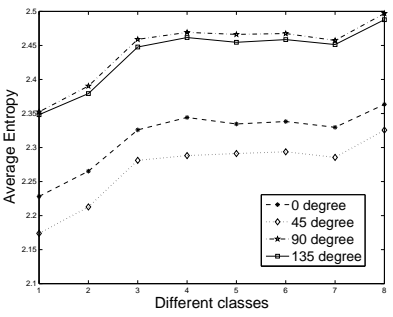

(a) Average Entropy

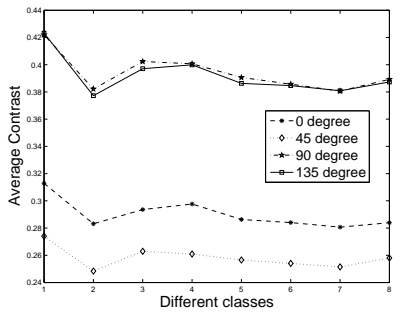

(c) Average Contrast

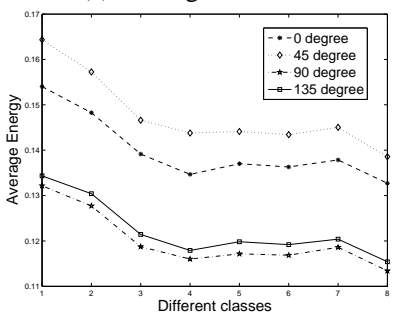

(e) Average Energy

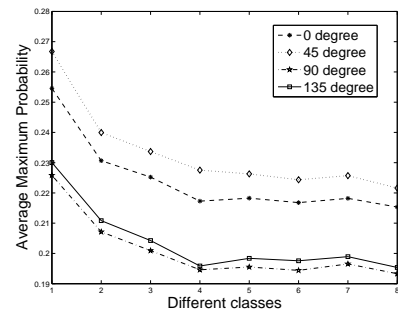

(b) Average Maximum Probability

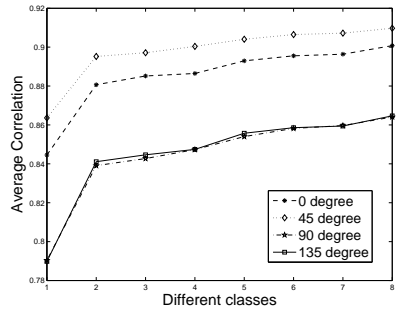

(d) Average Correlation

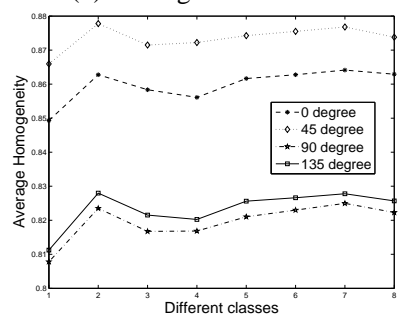

(f) Average Homogeneity
Fig. 3: Average GLCM features against class for the grey scale tea images, using quantisation level 8 , a single interpixel distance and four orientations $\left(0^{\circ}, 90^{\circ}, 45^{\circ}\right.$ and $\left.135^{\circ}\right)$.

\section{EXPERIMENTAL RESULTS}

\section{A. Results for PS moments}

We used the first four PS moments for the square and disk SE separately and then jointly as features, and employed all 
classifiers to classify the tea images according to granule size. For all classifiers, $70 \%$ of the sub-images from each class were randomly chosen as a training set and the remaining $30 \%$ subimages were used as a test set. The process was repeated 10 times and the results were averaged over the 10 runs.

Table I shows that the SVM attained $100 \%$ correct classification using the 4 PS moments computed from a disk SE, or a square SE, or both (using a linear kernel and cost of 1 or more for optimal results). The other classifiers produce lower error rates using the PS moments from the disk SE than the square SE. We also investigated using the moments from both the square and disk SEs, which gives similar results to the disk SE. (FF-NNET used 4, 10 and 10 hidden neurons respectively in the cases of the square SE, disk SE and both). Selection of an optimal SE depends on the geometric shapes we attempt to extract from the image. Tea granules are more likely to be a disk shape than square, so we would expect better classification using a disk SE.

TABLE I: Overall proportion of test images misclassified by all classifiers, using different sets of PS moments.

\begin{tabular}{|c|cccc|}
\hline SE & REG & SVM & LDA & FF-NNET \\
\hline Square & 0.214 & 0 & 0.018 & 0.019 \\
Disk & 0.123 & 0 & 0.009 & 0.012 \\
Square+Disk & 0.129 & 0 & 0.003 & 0.008 \\
\hline
\end{tabular}

Therefore we computed class-wise proportions of images misclassified, and MAE as in (4), for all classifiers using the 4 PS moments from the disk SE (Table II). The proportions misclassified and the MAEs are identical, as the predicted classes are at most one unit away from the actual class results. The SVM classifies perfectly, LDA is next best (a $0.9 \%$ error rate), then FF-NNET (1.3\% error rate), and then REG with a $9.9 \%$ error rate.

TABLE II: Class-wise and overall proportion of test images misclassified for all classifiers using 4 PS moments from a disk SE

\begin{tabular}{|c|cccc|}
\hline Class & \multicolumn{4}{|c|}{ Proportion misclassified } \\
\hline & REG & SVM & LDA & FF-NNET \\
\hline 1 & 0.000 & 0 & 0.000 & 0.000 \\
2 & 0.000 & 0 & 0.000 & 0.000 \\
3 & 0.020 & 0 & 0.000 & 0.000 \\
4 & 0.000 & 0 & 0.000 & 0.000 \\
5 & 0.100 & 0 & 0.000 & 0.000 \\
6 & 0.160 & 0 & 0.000 & 0.020 \\
7 & 0.220 & 0 & 0.030 & 0.046 \\
8 & 0.290 & 0 & 0.040 & 0.033 \\
\hline Overall & 0.099 & 0 & 0.009 & 0.013 \\
\hline
\end{tabular}

\section{B. Results for GLCM-based features}

We also used 4 GLCM features, i.e. entropy, maximum probability, correlation and energy, computed at quantisation 8 and $135^{\circ}$ orientation for classification. The same training strategy as above was applied for all classifiers (resulting in 8 hidden neurons in FF-NNET, and a linear SVM kernel with a cost of 1 or higher giving best results). Results are listed in Table III.

The MAE and proportion of test images misclassified are different in this case, as some of the predicted classes are more than one unit away from the actual classes. REG produced the highest MAE, and SVM the lowest. Again SVM is best, with no classification error, compared to $86.1 \%, 55.9 \%$ and $57.3 \%$ error rates respectively for REG, LDA and FF-NET. All except SVM are much poorer using the GLCM features than using the PS moments.

TABLE III: MAE and proportion of test images misclassified for all classifiers using 4 GLCM features.

\begin{tabular}{|c|cccc|}
\hline Class & \multicolumn{5}{|c|}{ MAE } \\
\hline & REG & SVM & LDA & FF-NNET \\
\hline 1 & 1.867 & 0 & 0.000 & 0.000 \\
2 & 2.360 & 0 & 0.967 & 1.173 \\
3 & 2.813 & 0 & 1.293 & 1.247 \\
4 & 3.213 & 0 & 0.747 & 0.933 \\
5 & 3.067 & 0 & 1.813 & 1.487 \\
6 & 2.947 & 0 & 1.693 & 1.460 \\
7 & 3.233 & 0 & 1.653 & 1.653 \\
8 & 2.826 & 0 & 1.233 & 1.640 \\
\hline Overall & 2.791 & 0 & 1.175 & 1.199 \\
\hline Class & \multicolumn{4}{|c}{ Proportion misclassified } \\
\hline & REG & SVM & LDA & FF-NNET \\
\hline 1 & 0.633 & 0 & 0.000 & 0.000 \\
2 & 0.607 & 0 & 0.353 & 0.427 \\
3 & 0.853 & 0 & 0.680 & 0.647 \\
4 & 0.980 & 0 & 0.413 & 0.540 \\
5 & 1.000 & 0 & 0.887 & 0.800 \\
6 & 0.987 & 0 & 0.920 & 0.807 \\
7 & 0.947 & 0 & 0.787 & 0.753 \\
8 & 0.880 & 0 & 0.433 & 0.613 \\
\hline Overall & 0.861 & 0 & 0.559 & 0.573 \\
\hline
\end{tabular}

\section{SUMmARY AND CONCLUSIONS}

This work provides greatly improved results of classifying these tea images compared to the results of [5], for all of the classifiers used. Our highest misclassification rate was for the regression approach $(9.9 \%)$, and the lowest was $0 \%$ for the SVM, using PS moments from the top-hat transformed images, whereas the error rates in [5] for the MLP and LVQ classifiers are $25.33 \%$ and $20 \%$ respectively. Their results are not exactly comparable to ours, as we have extracted our own subimages for algorithm development and testing. Nonetheless we conclude that extracting shape-based information from the tea granule images directly by use of morphological techniques provides very useful features for texture classification in any of a range of classifiers.

The key step in getting successful results from the PS moments for the tea images was to use the top-hat transform with a disk that increases in size for classes with larger tea granules. It was found that PS moments computed from the top-hat images obtained using the same size disk SE over all 8 classes produced very high classification error for most classifiers. For example, using a disk of radius 17, REG, SVM, LDA and FF-NNET using the same settings as before produced error rates of $90.5 \%, 0 \%, 63.4 \%$ and $70.3 \%$ 
respectively, whereas using increasing disk size in the top-hat transformation most of these classifiers achieved perfect or near perfect classification accuracy.

PS moments provide much better classification than GLCM features for these images. It was also found that a higher level of quantisation does not guarantee better classification results [27], as GLCM features computed at quantisation level 64 produced higher error rates for all classifiers except SVM.

\section{ACKNOWLEDGMENTS}

We are very grateful to E. Hines, S. Borah and M. Bhuyan for access to, and use of, the black tea granule images. Mahmuda Khatun was supported by a University of Strathclyde scholarship which made this work possible.

\section{REFERENCES}

[1] M.S. Zenoozian and S. Devahastin, Application of wavelet transform coupled with artificial neural network for predicting physicochemical properties of osmotically dehydrated pumpkin, Journal of Food Engineering, 90(2) (2009) 219-227.

[2] N. Bhattacharyya, S. Seth, B. Tudu, P. Tamuly, A. Jana, D. Ggosh, R. Bandyopadhyay, M. Bhuyan and S. Sabhapandit, Detection of optimum fermentation time for black tea manufacturing using electronic nose, Sensors and Actuators B, 122 (2007) 627-634.

[3] S. Borah and M. Bhuyan, Non-destructive testing of tea fermentation using image processing, Journal of Insight, 45 (2003) 55-58.

[4] J. Zhao, Q. Chen, J. Cai and Q. Quyang, Automated tea quality classification by hyper-spectral imaging, Journal of Applied Optics, 48(19) (2009) 3557-3564.

[5] S. Borah, E.L. Hines and M. Bhuyan, Wavelet transform based image texture analysis for size estimation applied to the sorting of tea granules, Journal of Food Engineering, 79(2) (2007) 629-639.

[6] W. Di, H. Yang, X. Chen, Y. He and X. Li, Application of image texture for the sorting of tea categories using multi-spectral imaging technique and support vector machine, Journal of Food Engineering, 88(4) (2008) 474-483.

[7] X-J. Chen, H-Q. Yang, D. Wu and Y. He, A new method to discriminate tea categories, Proceedings of the 7 th World Congress on Intelligent Control and Automation, Chongqing, China, (2008) 2236 - 2240.

[8] X-L. Li, Y. He and Z-j. Qiu, Textural feature extraction and optimization wavelet sub-bands for discrimination of green tea brands, Proceedings of the 7 th international Conference on Machine Learning and Cybernetics, Kunming, China, (2008) 1461-1466.

[9] G. Matheron, Random Sets and Integral Geometry, John Wiley and Sons, New York, 1975.

[10] J. Serra, Image Analysis and Mathemetical Morphology, Academic Press, London, 1983.

[11] H.J.A.M. Heijmans, Morphological Image Operators, Academic Press, Boston, 1994.

[12] E.R. Dougherty and J. Astola, An Introduction to Nonlinear Image Processing, SPIE Press, Washington, USA, 1994.
[13] E.R. Dougherty and R.A. Lotufo, Hands-on Morphological Image Processing, SPIE Press, Washington, USA, 2003.

[14] J. Goutsias, H.J.A.M. Heijmans and K. Sivakumar, Morphological operators for image sequences, Journal of Computer Vision and Image Understanding, 62(3) (1995) 326-346.

[15] A. Mavilio, M. Fernández, M. Trivi, H. Rabal and R. Arizaga, Characterization of a paint drying process through granulometric analysis of speckle dynamic patterns, Signal Processing, 90(5) (2010) 1623-1630.

[16] M.E. Díaz, G. Ayala, R. Sebastian and L. Martínez-Costa, Granulometric analysis of corneal endothelium specular images by using a germ and grain model, Computers in Biology and Medicine, 37(3) (2007) 364-375.

[17] N. Theera-Umpon and S. Dhompongsa, Morphological granulometric features of nucleus in automatic bone marrow white blood cell classification, IEEE Transactions on Information Technology in Biomedicine, 11(3) (2007) 353-359.

[18] A.J. Gray, S. Marshall, and J. McKenzie, Modeling of evolving textures using granulometries, In Marshall, S. and Sicuranza, G.L. (eds.), Advances in Nonlinear Signal and Image Processing, EURASIP Series on Signal Processing and Communications, Hindawi Publishing Corporation, New York, 2006.

[19] Y. Chen and E.R. Dougherty, Grey scale morphological granulometric texture classification, Optical Engineering, 33(8) (1994) 2713-2722.

[20] P. Brodatz, Textures: A Photographic Album for Artists and Designers, Dover, New York, 1966.

[21] W.D. Carlos, M.R.C. Almeida and L.B.C. Ana, Texture classification based on co-occurrence matrix and self-organizing map, IEEE International Conference on Systems, Man and Cybernatics, Istanbul, (2010) 2487-2491.

[22] J.K. Shuttleworth, A.G. Todman, R.N.G. Naguib, B.M. Newman, and M.K. Bennett, Colour texture analysis using co-occurrence matrices for classification of colon cancer images, IEEE Canadian Conference on Electrical and Computer Engineering, Canada, (2002) 1134-1139.

[23] C. Palm, Color texture classification by integrative co-occurrence matrices, Pattern Recognition, 37(5) (2004) 965-976.

[24] M. Masotti and R. Campanini, Texture classification using invariant ranklet features, Pattern Recognition Letters, 29(14) (2002) 1980-1986.

[25] R.C. Gonzalez and R.E. Woods, Digital Image Processing, Third Edition, Prentice Hall, New Jersey, 2008.

[26] P. Maragos, Pattern spectrum and multiscale shape representation, IEEE Transactions on Pattern Analysis and Machine Intelligence, 11(7) (1989) 701-716.

[27] D.A. Clausi, An analysis of co-occurrence texture statistics as a function of grey level quantization, Canadian Journal of Remote Sensing, 28(1) (2002) 45-62.

[28] R.M. Haralick, K. Shanmugan, and I. Dinstein, Textural features for image classification, IEEE Transactions on Systems, Man and Cybernetics, 3(6) (1973) 610-621.

[29] C. Cortes and V. Vapnik, Support-vector networks, Machine Learning, 20(3) (1995) 273-297.

[30] A.J. Izenman, Modern Multivariate Statistical Techniques: Regression, Classification, and Manifold Learning, Springer, USA, 2008.

[31] B. Zhang, Y. Song, S. Guan and Y. Zhang, Historic Chinese architectures image retrieval by SVM and pyramid histogram of oriented gradient features, International Journal of Soft Computing, 5(2) (2010) 19-28.

[32] D.J. Hand, Discrimination and Classification, John Wiley and Sons Ltd, Chichester, UK, 1981 